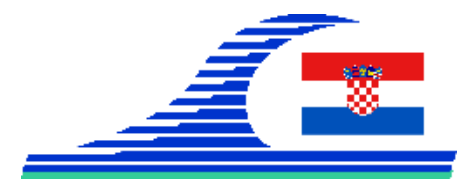

\author{
Conférence Méditerranéenne Côtière et Maritime \\ EDITION 4, SPLIT, CROATIA (2017) \\ Coastal and Maritime Mediterranean Conference \\ Disponible en ligne - http://www.paralia.fr - Available online
}

\title{
Les interventions d'urgence sur les dispositifs de protection contre les submersions marines Un nouveau guide aux Éditions du CEREMA
}

\section{Marc IGIGABEL $^{1}$}

1. CEREMA, Technopôle Brest Iroise, 155 rue Pierre Bouguer, BP5, 29280 Plouzané, France.

marc.igigabel@cerema.fr

\section{Résumé :}

Si les interventions sur les ouvrages de protection contre les submersions marines doivent en général faire l'objet d'études précises et complètes, les interventions en situation d'urgence sont soumises à la contrainte du temps qui impose d'identifier rapidement les solutions techniques les plus pertinentes et de les mettre en œuvre dans un délai également contraint. Dans le domaine côtier, le passage des tempêtes, les variations du niveau marin, les vagues et les courants confèrent un rythme particulier à ces opérations. Par ailleurs, les particularités des ouvrages côtiers ne peuvent être prises en compte que par des techniques adaptées. Ces spécificités ont justifié de consacrer un guide sur le sujet (CEREMA, à paraître). Les recommandations formulées dans ce guide reposent sur un principe fondamental : une intervention d'urgence doit être anticipée (stratégie, préparation, entrainement), évaluée et améliorée continuellement. Pour répondre au caractère opérationnel des interventions d'urgence, les deux premiers chapitres du guide traitent distinctement :

- des actions qui s’inscrivent dans le « temps long » et qui correspondent à la définition de l’organisation générale, basée sur des phases d'études et de tests et matérialisée par la production et la mise à jour d'un plan d'intervention,

- des actions qui s’inscrivent sur des «temps courts » : la réservation de matériel et la constitution de stocks de matériaux, les entraînements et les exercices, les actions de surveillance, les interventions sur les digues, les ouvrages hydrauliques et les réseaux, l'adaptation des actions à l'évolution de la situation.

Dans un troisième chapitre sont exposées les techniques d'intervention sur les ouvrages. Ces préconisations sont organisées en relation avec les «modes de défaillance » des ouvrages. Elles visent à éclairer la prise de décision en réponse à des situations typiques.

\section{Mots-clés :}

Interventions, Urgence, Protection, Submersions marines, Système d'endiguement, Techniques. 
Mediterranean rocky coasts:

Features, processes, evolution and problems

\section{Introduction}

Le 28 février 2010, la tempête Xynthia a rappelé la grande vulnérabilité de notre littoral au risque de submersion par la mer. Elle a également révélé un manque de préparation à deux niveaux : d'une part, le risque d'inondation par submersion marine n'a pas été correctement évalué dans la préparation à la crise (BERSANI et al., 2010) ; d'autre part, le manque de préparation sur le plan opérationnel a conduit à un allongement des temps d'intervention sur les ouvrages de protection et à des choix techniques apparaissant souvent, a posteriori, peu pertinents. En réponse à cette situation, la stratégie nationale de gestion des risques d'inondation et la stratégie de gestion du trait de côte constituent aujourd'hui la base des politiques publiques d'évaluation et de prévention des risques de submersion marine. Ces stratégies préconisent une approche globale des enjeux et des aléas de submersions. Leur mise en œuvre au niveau local doit néanmoins s’appuyer sur des documents techniques de référence. Pour accompagner les organismes en charge de ces actions, des guides à vocation méthodologique ont ainsi été produits au cours des dernières années, notamment le guide pour l'élaboration des plans de prévention des risques littoraux (DGPR/CEREMA, 2014) et le guide relatif aux Plans communaux de sauvegarde (DDSC, 2005). Les retours d'expérience constituant un matériau privilégié pour améliorer les mesures de prévention, une méthodologie portant sur le diagnostic des systèmes de protection contre les submersions marines (CEREMA, 2016) a également été élaborée à la suite de Xynthia. En complément, concernant le sujet des interventions d'urgence sur les ouvrages de protection, il est apparu nécessaire de produire une nouvelle référence s’appuyant sur des retours d'expériences récents. C’est tout l'objet du guide (CEREMA, à paraître) présenté dans cet article.

\section{Objectifs du guide}

Cette nouvelle référence est destinée à apporter une aide dans la préparation et la réalisation des interventions sur les dispositifs de protection contre les submersions marines en période de crise. (Le guide ne décrit pas les autres mesures qui doivent être prises vis-à-vis de la population et de la protection des biens.) Le guide est rédigé suivant le principe qu'une intervention d’urgence doit être anticipée, évaluée et améliorée continuellement par l'élaboration et la mise à jour d'un plan d'intervention. Bien que toutes les circonstances d'une crise ne puissent être anticipées, l'élaboration d'un tel plan améliorera les capacités de réaction à des situations variées. (A contrario, le manque d'anticipation augmente le risque de prendre des décisions hasardeuses.)

Les interventions d'urgence considérées concernent l'ensemble des actions de gestion de crise qui peuvent être entreprises afin d'influer sur les écoulements d'eau. La période d'intervention considérée court de l'annonce de la tempête jusqu'à la fin des opérations d'urgence visant à rétablir un niveau de protection acceptable pour faire face à un nouvel événement. Si les eaux ont envahi un territoire la période considérée couvre également les opérations de ressuyage. 
Mediterranean rocky coasts:

Features, processes, evolution and problems

Les ouvrages concernés sont principalement :

- les digues (inspections et diagnostics, travaux de confortement, réparation ou comblement de brèche),

- les ouvrages hydrauliques et les réseaux d'assainissement, ainsi que les dispositifs temporaires de retenue d'eau et les systèmes de pompage de secours.

Si dans le domaine côtier les dunes peuvent participer à la protection contre les inondations, ce sont des éléments qui impliquent de prendre en compte des aspects environnementaux particuliers, tant sur le plan des processus physiques et naturels que sur le plan des réglementations environnementales. Les dunes sont donc hors du champ des préconisations et des méthodes présentées dans ce guide. Le gestionnaire de digues doit toutefois intégrer les dunes et autres formations naturelles dans le périmètre de ses réflexions comme un élément de contexte important.

\section{Contenu et forme du guide}

Le guide comprend trois parties :

- Partie 1: le cadre stratégique des interventions d'urgence sur les ouvrages. La définition de ce cadre - qui se traduit par l'établissement d'un plan de gestion des ouvrages - implique :

- de connaître certaines règles communes à tous les systèmes d'endiguement pour l’organisation de la préparation et de la réponse,

- de mener certaines études pour adapter le plan de gestion des ouvrages aux conditions météorologiques et hydrauliques, au système de protection et aux enjeux exposés, ainsi qu'au risque de défaillance des ouvrages,

- Partie 2 : les actions particulières de préparation et de gestion de crise, dont :

- la réservation de matériel et la constitution de stocks de matériaux,

- la formation et l'entraînement du personnel mobilisé,

- des actions de surveillance, d'exploitation et de réparation des ouvrages,

- l'adaptation des actions à l'évolution de la situation,

- Partie 3 : les principales techniques d'intervention sur les ouvrages. Ces préconisations sont organisées en relation avec les «modes de défaillance »:

- protection contre l'érosion externe,

- protection contre l'érosion interne,

- protection contre les instabilités,

- comblement des brèches et autres réparations urgentes destinées à remédier aux insuffisances des ouvrages,

- réalisation de dispositifs temporaires de retenue d'eau (disposition acceptable dans des conditions bien déterminées),

- les systèmes de fermeture des réseaux d'assainissement, les systèmes de pompage de secours. 
Mediterranean rocky coasts:

Features, processes, evolution and problems

\section{Conclusions}

Une intervention d'urgence sur un ouvrage de protection contre les submersions marines est décidée au regard du risque de mise en danger des populations et des enjeux vulnérables. Le mode d'intervention est ensuite conditionné par de nombreux paramètres : type d'ouvrage, position et fonction de l'ouvrage dans le système de protection, conditions hydrauliques, conditions d'accès, état présent et évolution de l'ouvrage, techniques envisageables, moyens et matériaux disponibles pour les mettre en œuvre, temps nécessaire d'intervention, réversibilité de l'opération, impact sur l'environnement, coût... Rédigé sur la base du retour d'expérience Xynthia et de la littérature étrangère, notamment (CIRIA, CETMEF, USACE, 2013), le guide (CEREMA, à paraître) apporte aux gestionnaires des systèmes d'endiguement côtiers des repères en période de préparation et en période de crise. En complément des éléments méthodologiques et techniques, les dispositions qu'impose la réglementation en matière d'intervention sur les systèmes d'endiguement sont rappelées pour mieux préciser le contexte français. Signalons en particulier qu'en France, la réalisation en urgence de dispositifs de retenue d'eau ne peut être entreprise que très ponctuellement, pour le traitement de points bas apparaissant sur des digues existantes ou alors pour la création ex nihilo d'ouvrages temporaires destinés à la protection d'enjeux particuliers.

Dans la perspective d'un changement climatique dont la surélévation du niveau marin est un des effets les plus certains, l'adaptation des dispositifs de protection contre les submersions marines est et restera longtemps un enjeu majeur des politiques de prévention des risques. Les recommandations proposées dans le guide (CEREMA, à paraître) visent donc à contribuer sur le long terme à la définition des stratégies de protection en apportant un meilleur éclairage sur les capacités réelles d’intervention en période de crise.

\section{Références bibliographiques}

BERSANI C., DUMAS P., ROUZEAU M., GERARD F., GONDRAN O., HELIAS A., MARTIN X., PUECH P., FLEURY B., GREFF M., BOUGERE R., TREPOS Y. (2010). Tempête Xynthia, retour d'expérience, évaluation et propositions d'action. Ministère de l'écologie, de l'énergie, du développement durable et de la mer, Rapport, 191p.

CEREMA. (à paraître). Interventions d'urgence sur les dispositifs de protection contre les submersions marines

CEREMA. (2016). Étude des systèmes de protection contre les submersions marines Méthodologie et études de cas issues du retour d'expérience Xynthia. CEREMA Editeur, 446p. ISBN 978-2-37180-118-9

CIRIA, CETMEF, USACE. (2013). The International Levee Handbook, C731, CIRIA, London, ISBN: 978-0-86017-734-0 
Mediterranean rocky coasts: Features, processes, evolution and problems

DDSC.- Direction de la Défense et de la Sécurité Civiles - (2005). Plan communal de sauvegarde - Guide pratique d'élaboration. Ministère de l'intérieur et de l'aménagement du territoire, 202p.

DGPR/CEREMA. (2014). Guide Méthodologique Plan de Prévention des risques littoraux. MEDDE, 169p. 
Mediterranean rocky coasts:

Features, processes, evolution and problems 